INRA Prod. Anim.,

2012, 25 (2), 211-220

\title{
Le travail, sujet intime et multifacette : premières recommandations pour l'aborder dans le conseil en élevage
}

\author{
F. KLING-EVEILLARD 1 , M. CERF ${ }^{2}$, S. CHAUVAT ${ }^{3}$, N. SABATTE 4 \\ ${ }^{1}$ Institut de l'Elevage, 149 rue de Bercy, F-75595 Paris, France \\ 2 INRA, UR 1326 Sens, Bâtiment EGER, BP 1, F-78850 Thiverval Grignon, France \\ ${ }^{3}$ Institut de l'Elevage, SupAgro, 2 Place Pierre Viala, F-34060 Montpellier, France \\ ${ }^{4}$ Chambre d'Agriculture de la Savoie, 40 rue du Terraillet, F-73190 St Baldoph, France \\ Courriel : florence.kling@idele.fr
}

Le travail constitue un enjeu essentiel pour les éleveurs. S'il est de plus en plus pris en compte dans le conseil, diverses études montrent néanmoins que les conseillers éprouvent des difficultés à l'inscrire dans leur activité. L'analyse de ces difficultés permet de formuler des recommandations pratiques pour que les spécificités du sujet du travail soient mieux prises en compte dans l'accompagnement des éleveurs.

Le travail constitue un enjeu majeur en élevage parce qu'il joue directement sur la «vivabilité» des exploitations et donc sur leur pérennité. Les évolutions structurelles en cours, telles que l'agrandissement, la réduction de la maind'œuvre familiale, l'accroissement de la productivité par travailleur, contribuent souvent à une dégradation des conditions de travail. Par ailleurs, dans les exploitations sociétaires en plein développement, la conduite et le management deviennent plus complexes et l'enjeu lié au travail y est exacerbé. Enfin, les éleveurs eux-mêmes mettent en avant aujourd'hui de nouvelles visions de leur métier (Couzy et Dockès 2006) et expriment de plus en plus souvent des aspirations en termes d'évolution de leurs conditions de travail, afin de disposer de temps libéré pour des loisirs ou d'autres activités.

L'offre de conseil sur le travail en élevage se structure progressivement (Kling-Eveillard et al 2007). Ainsi, des conseillers sont identifiés comme personnes ressources sur le sujet dans les organismes de développement; des démarches d'accompagnement avec leurs outils sont élaborées et mises à disposition du terrain; des accompagnements individuels ou en groupe (formations ou réunions) sont proposés aux éleveurs. A l'échelle de territoires, des actions concertées se mettent en place pour articuler les compétences et les intervenants sur le travail en élevage (Kling-Eveillard et al 2010).
De façon paradoxale, le travail reste cependant un sujet dont les éleveurs parlent relativement peu, que ce soit entre eux ou avec un conseiller, et qui suscite de fait peu de demandes spontanées d'accompagnement et peu d'adhésion aux actions telles que celles citées plus haut quand elles sont proposées sur le terrain. Les conseillers disent fréquemment qu'il est difficile de mobiliser les éleveurs sur cette question qui constitue pourtant l'une de leurs principales préoccupations. Ils sont nombreux à faire part de leurs propres difficultés à s'approprier le sujet et à se positionner comme ressource sur ce thème auprès des éleveurs.

Nous nous intéressons dans cet article à ce que les conseillers vivent quand ils mettent en œuvre dans un élevage un conseil sur le travail. Nous nous proposons, à partir d'expériences de terrain, d'analyser les difficultés que les conseillers ressentent et expriment, en vue de formuler des recommandations pour lever ces difficultés.

\section{1 / Se doter d'un cadre pour valoriser des études de ter- rain abordant le conseil sur le travail}

Formuler des recommandations en matière de conseil à partir des difficultés énoncées par les conseillers, nécessite de se doter d'un cadre d'analyse pour les structurer et déboucher alors sur des suggestions, visant à les dépasser tout en conservant certaines caractéristiques des formes d'exercice du conseil sur le travail en élevage. Avant cela, nous nous proposons de définir la façon dont nous envisageons le travail comme objet de conseil.

\section{1 / Le travail comme objet d'un conseil en élevage}

Le terme travail peut renvoyer à des objets appréhendés à différentes échelles, qu'il s'agisse de l'activité, de la profession, de la tâche. Le travail s'exerce dans des rapports au monde matériel et vivant, dans lesquels l'individu engage son corps (dimension physique) et sa personne (dimensions identitaire et sociale, affective et cognitive). Celui ou celle qui travaille «fait des choses en travaillant» mais tout autant se construit personnellement ou socialement (Marx cité par Méda 2007). C'est sans doute encore plus prégnant en agriculture, car le travail agricole présente entre autres particularités, celle d'être très imbriqué avec la vie familiale et personnelle et de s'articuler avec les projets de vie des agriculteurs (Béguin et al 2011). Cela rend parfois difficile l'identification de ce que recouvre la notion de travail pour une personne donnée et la manière dont ce sujet peut être abordé dans un conseil. Se posent ainsi des questions sur la façon dont le travail peut être d'une part, analysé et d'autre part, faire l'objet d'un conseil. 
Dedieu et Servière (2011) ont formalisé trois modèles qui sous-tendent les démarches d'analyse du travail dans les systèmes d'élevage et les démarches d'accompagnement des éleveurs : le «travail ressource» considéré comme un facteur de production à optimiser, le «travail organisé» considéré comme un système complexe d'activités articulées dans le temps et le «travail subjectivé» centré sur l'homme et sur les raisons subjectives du «travailler» (décrites par Fiorelli et al 2010). Ainsi, d'après ces auteurs, trois angles peuvent être adoptés pour traiter du travail dans les élevages : chercher à optimiser l'utilisation du temps ou la productivité, s'attacher à améliorer l'organisation au sein d'un collectif ou la coexistence d'activités diverses sur l'exploitation, ou encore interroger le sens du métier, source de plaisir et déplaisir au travail.

En pratique, dans l'activité de conseil, le travail est souvent décliné selon plusieurs dimensions parmi lesquelles : le volume global, la charge quotidienne et l'astreinte (en particulier en lien avec les soins aux animaux), la répartition du temps de travail sur la semaine et les week-ends, la répartition des tâches sur l'année et les variations quantitatives (dont les périodes de pointe) et qualitatives (congés, travaux différents), la combinaison des activités (élevage et autres en particulier), la répartition des tâches au sein du collectif, les relations humaines, la pénibilité physique, la pénibilité mentale et le stress, les horaires sur la journée, la santé et la sécurité au travail, etc. Ces dimensions s'inscrivent bien dans les trois modèles explicités par Dedieu et Servière (2011) tout en les abordant de façon éventuellement partielle. Par ailleurs, elles mobilisent des connaissances issues de disciplines aussi variées que la zootechnie, la sociologie, la gestion et l'économie, l'ergonomie, le management, etc. Une dimension donnée peut ellemême nécessiter des regards croisés : ainsi, la sécurité et la santé au travail peuvent mobiliser la médecine et l'ergonomie.

De ce qui précède, nous retenons l'idée que le travail est à la fois un sujet «intime» et un sujet multifacette dans la façon de l'aborder dans les systèmes d'élevage d'un point de vue analytique ou de conseil. Ces caractéristiques sontelles sources de difficultés pour les conseillers ? Comment les vivent-ils et comment éventuellement les dépassentils?
1.2 / La relation de conseil : une approche interactionniste resituee dans les étapes du conseil

Pour répondre à ces questions, il nous paraît important d'étudier comment les conseillers énoncent les effets des caractéristiques du travail sur leur relation avec l'éleveur. Nous proposons pour cela d'articuler le cadre d'interaction avec un «client» proposé par Cerf et Falzon (2005) avec une analyse des étapes qui permettent de passer d'une demande d'un conseil sur le travail à l'élaboration d'un plan d'action pour résoudre un problème.

Rappelons tout d'abord ce que conseiller veut dire. Dans la théorie des actes de langage, le conseil est défini comme ce qui concerne l'orientation d'une action future de celui qui est conseillé, dans l'intérêt de celui-ci (Mayen, communication personnelle). Mais cette définition, centrée sur la façon dont s'exprime le conseiller, doit être resituée dans un cadre interactionniste plus large dans le prolongement des travaux de Goffman (1968). Ainsi, Cerf et Falzon (2005) distinguent trois niveaux qui interfèrent dans la réussite de la relation entre un professionnel (le conseiller) et un bénéficiaire (un ou des éleveurs) : le niveau technique ou transactionnel (en lien avec la problématique à traiter dans le conseil, c'est-à-dire avec l'objet que constitue le travail), le niveau interactionnel (autour des modalités et des rôles de chacun dans l'échange) et le niveau contractuel (prenant en compte les relations institutionnelles et les positions des acteurs). Ce cadre va nous permettre de comprendre comment les spécificités du sujet du travail (caractères intime et multifacette) engendrent pour les conseillers des écueils dans la conduite des interactions. Nous pourrons alors étudier plus précisément les difficultés opérationnelles qu'ils rencontrent aux différentes étapes classiquement identifiées du processus de conseil : l'étape d'émergence et d'accueil de la demande, celle de la formulation du problème et du diagnostic, et celle de la réflexion autour de solutions ou de la construction d'un plan d'amélioration.

Le cadre d'analyse choisi nous sert à lire a posteriori des études qui ont été réalisées (tableau 1) pour décrire des actions concrètes et recueillir le vécu des conseillers. Il n'a donc pas orienté le recueil des données mais il contribue plutôt à donner une certaine intelligibilité transversale à ces différentes études de cas pour produire des recommandations quant à la façon de dépasser les difficultés énoncées par les conseillers.

\section{2 / Capitaliser les enseigne- ments du terrain et le vécu des conseillers}

Il n'existe pas d'étude exhaustive sur la mise en œuvre d'un conseil sur le travail en élevage qui permettrait de disposer d'une vue d'ensemble de la diversité des modes d'intervention, des profils de conseillers et de leur vécu du conseil. Nous nous appuyons dans notre réflexion sur plusieurs travaux portant sur des actions d'accompagnement des éleveurs sur le sujet du travail, sur une analyse de la mise en œuvre d'outils de conseil sur le travail, ainsi que sur la synthèse d'actions de terrain par les conseillers qui les ont mises en œuvre. Ces travaux ont été réalisés dans le cadre du Réseau Mixte Technologique (RMT) «Travail en élevage». Les actions analysées s'inscrivent dans des contextes régionaux où la question du travail en élevage est posée comme un enjeu important et mobilise des moyens humains et financiers spécifiques. Les points de vue des conseillers dans ces situations ne peuvent donc être considérés comme représentatifs de ceux de l'ensemble des conseillers sur le travail en élevage au niveau français, mais ils en couvrent cependant une diversité suffisante pour constituer le support d'une synthèse et autoriser, en nous concentrant sur des résultats convergents, à formuler des recommandations à valeur générale.

Les études analysées (tableau 1) portent principalement sur l'évaluation de l'impact d'un conseil sur le travail en élevage auprès des éleveurs, des conseillers et des partenaires des actions. Ces travaux ont été conduits à différentes échelles dans le cadre soit d'accompagnements individuels d'éleveurs (expérimentation conseil pluridisciplinaire Pays de la Loire : Rubin et Sabatté 2008, Sabatté et Huchon 2009), soit d'actions régionales (évaluations «Paqvel» ${ }^{1}$ en Aquitaine : Marty 2004, Roussez 2008 ; «Vip» ${ }^{2}$ en Picardie : Kling-Eveillard et al 2010, Warcoin 2010), soit de mise en œuvre d'outils de conseil (outils de diagnostic «Actel» ${ }^{3}$ en élevage laitier et Conseil Travail en élevage ovin, pré diagnostic «Arefa» ${ }^{4}$ en

\footnotetext{
1 Paqvel : Programme d'amélioration de la qualité de vie en élevage laitier (Aquitaine).

2 Vip : Vivre l'élevage en Picardie, action régionale qui comporte un volet «Travail et qualité de vie».

3 Actel : Amélioration des conditions de travail en élevage laitier.

4 Arefa : Association régionale emploi formation en agriculture.
} 
Tableau 1. Caractéristiques des études supports de l'analyse du conseil sur le travail en élevage.

Quand l'étude ne concerne que certaines filières d'élevage celles-ci sont indiquées.

\begin{tabular}{|c|c|c|}
\hline $\begin{array}{l}\text { Nom de l'étude } \\
\text { et références }\end{array}$ & Objectifs & $\begin{array}{l}\text { Méthode de recueil des avis } \\
\text { et caractéristiques des conseillers enquêtés }\end{array}$ \\
\hline $\begin{array}{l}\text { Evaluation de l'action } \\
\text { régionale "Paqvel» } 2004 \\
\text { Marty (2004) }\end{array}$ & $\begin{array}{l}\text { - Evaluation de la satisfaction des partenaires } \\
\text { de l'action de conseil collectif et de leur } \\
\text { potentiel d'implication pour la suite } \\
\text { - Evaluation de la satisfaction des éleveurs } \\
\text { bovins laitiers ayant participé aux premières } \\
\text { réunions «Paqvel» } \\
\text { - Proposition de pistes d'amélioration }\end{array}$ & $\begin{array}{l}\text { - Entretiens semi-directifs en face-à-face } \\
\text { avec } 24 \text { partenaires : CA, OCEL, Cuma, } \\
\text { laiteries, syndicalisme, services de } \\
\text { remplacement, centres de gestion, MSA, } \\
\text { Aract }\end{array}$ \\
\hline $\begin{array}{l}\text { Evaluation «Paqvel» } 2008 \\
\text { Roussez (2008) }\end{array}$ & $\begin{array}{l}\text { - Evaluation des effets de l'action de conseil } \\
\text { collectif : satisfaction et pertinence pour les } \\
\text { conseillers et les éleveurs } \\
\text { - Proposition de pistes d'amélioration }\end{array}$ & $\begin{array}{l}\text { - Entretiens semi-directifs avec } 10 \\
\text { conseillers (CA, laiteries, OCEL, Services } \\
\text { de remplacement) }\end{array}$ \\
\hline $\begin{array}{l}\text { Expérimentation d'un } \\
\text { conseil pluridisciplinaire en } \\
\text { Pays de Loire } \\
\text { Rubin et Sabatté (2008), } \\
\text { Sabatté et Huchon (2009) }\end{array}$ & $\begin{array}{l}\text { - Evaluation de l'impact d'accompagnements } \\
\text { individuels auprès d'éleveurs bovins laitiers } \\
\text { - Bilan par les conseillers impliqués } \\
\text { - Proposition d'une démarche } \\
\text { d'accompagnement de la réflexion sur le } \\
\text { travail }\end{array}$ & $\begin{array}{l}\text { - Entretiens semi-directifs en face-à-face et } \\
\text { réunions de travail avec: } \\
\text { - } 14 \text { conseillers d'entreprise CA dont } 4 \\
\text { identifiés sur le conseil (travail») } \\
\text { - } 17 \text { conseillers techniques spécialisés CA } \\
\text { (lait, traite, bâtiment, paysage...) } \\
\text { - } 4 \text { consultants (ergonomie, relations } \\
\text { humaines, analyse stratégique) }\end{array}$ \\
\hline $\begin{array}{l}\text { Analyse de la mise en } \\
\text { cuvre d'outils de conseil } \\
\text { sur le travail } \\
\text { Renard (2009) }\end{array}$ & $\begin{array}{l}\text { - Evaluation des facteurs déterminants de } \\
\text { P'appropriation des outils «Actel»\} (élevages } \\
\text { laitiers), Conseil Travail en élevage Ovin, } \\
\text { pré diagnostic "Arefa» } \\
\text { - Typologie des utilisateurs d'outils } \\
\text { - Formulation de recommandations pour } \\
\text { l'organisation du conseil sur le travail à } \\
\text { l'échelle d'un territoire }\end{array}$ & $\begin{array}{l}\text { - Entretiens semi-directifs en face-à-face } \\
\text { avec: } \\
\text { - } 15 \text { conseillers (CA, OP, OCEL) } \\
\text { - } 10 \text { responsables de service et } \\
\text { animateurs (dont des concepteurs des } \\
\text { outils évalués) }\end{array}$ \\
\hline $\begin{array}{l}\text { Evaluation de l'action } \\
\text { régionale "Vip» } 2010 \\
\text { Kling-Eveillard et al (2010), } \\
\text { Warcoin (2010) }\end{array}$ & $\begin{array}{l}\text { - Evaluation de l'impact de l'action régionale } \\
\text { (dont la preparation des actions du volet } \\
\text { Travail et qualité de vie) : satisfaction et } \\
\text { pertinence pour les partenaires et les } \\
\text { éleveurs (bovins lait ou viande, ovins } \\
\text { viande) } \\
\text { - Proposition de pistes d'amélioration }\end{array}$ & $\begin{array}{l}\text { - Entretiens semi-directifs en face-à-face ou } \\
\text { par télephone avec } 20 \text { conseillers (OCEL, } \\
\text { CA, centre de gestion, laiterie...) }\end{array}$ \\
\hline $\begin{array}{l}\text { Bilan de } 6 \text { expériences } \\
\text { d'accompagnement } \\
\text { d'éleveurs sur le travail } \\
\text { Kling-Eveillard et af (2008) }\end{array}$ & $\begin{array}{l}\text { - Description factuelle de l'expérience de } \\
\text { terrain (contexte, démarche et etat } \\
\text { d'avancement, organisation, partenariat, } \\
\text { réalisations) } \\
\text { - Bilan par l'animateur, quelques partenaires } \\
\text { et parfois quelques eleveurs (points positifs, } \\
\text { points sensibles, recommandations et } \\
\text { perspectives d'avenir) }\end{array}$ & $\begin{array}{l}\text { - Entretiens téléphoniques pour les } \\
\text { animateurs de l'Afocg de l'Ain, de l'Arefa } \\
\text { Midi-Pyrénées, de la CA du Loir-et-Cher } \\
\text { (Gehodes), } \\
\text { - Entretiens en face-à-face pour les } \\
\text { expériences des Chambres d'agriculture } \\
\text { de Normandie, de l'Ede du Puy-de-Dôme, } \\
\text { du Parc Naturel du Pilat }\end{array}$ \\
\hline $\begin{array}{l}\text { Recensement de } 60 \text { actions } \\
\text { d'accompagnement des } \\
\text { éleveurs sur le travail } \\
\text { Kling-Eveillard et al ( } 2007)\end{array}$ & $\begin{array}{l}\text { - Description factuelle par les maîtres d'œuvre } \\
\text { (type d'action, objectifs operationnels, } \\
\text { public, partenaires, durée prévue, état } \\
\text { d'avancement) } \\
\text { - Points forts et difficultés rencontrés, } \\
\text { propositions pour améliorer le conseil sur le } \\
\text { travail }\end{array}$ & $\begin{array}{l}\text { - Questionnaire écrit rempli par les maîtres } \\
\text { d'œuvre ou animateurs des } 60 \text { actions } \\
\text { recensées }\end{array}$ \\
\hline $\begin{array}{l}\text { Atelier d'échanges des } 3^{\text {èmes }} \\
\text { Rencontres Nationales du } \\
\text { RMT Travail } \\
\text { Fiorelli et Couzy (2009), } \\
\text { Gédouin et Guillaumin (2009), } \\
\text { Tellier et Chauvat (2009) }\end{array}$ & $\begin{array}{l}\text { - Confrontation d'expériences de conseillers } \\
\text { autour du conseil sur le travail en élevage et } \\
\text { réflexion collective sur des } \\
\text { recommandations pour la mise en ceuvre }\end{array}$ & $\begin{array}{l}\text { - } 3 \text { groupes d'une vingtaine de participants } \\
\text { - } 3 \text { thèmes : atelier } 3 \text { «Actions partenariales } \\
\text { d'accompagnement des éleveurs»; atelier } \\
4 \text { «Evolution du métier de conseiller»; } \\
\text { atelier } 7 \text { «Partage d'expériences sur les } \\
\text { façons d'être» } \\
\text { - } 2 \text { à } 3 \text { exposés introductifs sur une } \\
\text { expérience de terrain puis réflexion } \\
\text { collective }\end{array}$ \\
\hline
\end{tabular}

CA : Chambre d'Agriculture ; OP : Organisation de Producteurs ; OCEL : Organisme de Contrôle Laitier ; Cuma : Coopérative d'utilisation de matériel en commun; MSA : Mutualité Sociale Agricole; Afocg: Association pour la formation collective à la gestion ; Aract : Association régionale pour l'amélioration des conditions de travail ; Paqvel : Programme d'amélioration de la qualité de vie en élevage laitier en Aquitaine ; Actel : Amélioration des conditions de travail en élevage bovin lait ; Arefa : Association régionale emploi formation en agriculture ; Vip : Vivre l'élevage en Picardie ; EDE : Etablissement Départemental de l'Elevage. 
agriculture : Renard 2009). Ils mettent en œuvre des approches qualitatives qui portent sur les représentations sociales des acteurs et leurs pratiques à des fins de compréhension de ce qu'ils ont vécu ou mis en œuvre lors de cette activité de conseil en élevage, en complément de la description des moyens (dispositifs et fonctionnement, acteurs impliqués, méthodes et outils...). Les réalisations et les résultats sont appréciés à travers le nombre et les caractéristiques des éleveurs concernés, les accompagnements effectués, les supports d'information diffusés, les réunions d'échanges et les formations des agents de terrain réalisées, etc.

Au cours de l'évaluation de ces dispositifs de conseil, des entretiens qualitatifs de type semi-directif en face-à-face, selon la méthode décrite par Blanchet et Gotman (1992) ont été menés auprès de conseillers et pour certains travaux auprès d'éleveurs. Ils ont été conduits sur la base d'échantillons limités en nombre mais raisonnés de façon à couvrir la diversité des formes de conseil (conseil individuel, de groupe...) qui ont été proposées aux éleveurs. Les entretiens ont été enregistrés, transcrits et dépouillés selon les méthodes d'analyse de contenu thématique classiquement mises en œuvre en sociologie ou en psychologie sociale (Ghiglione et Matalon 2008). Au cours de ces entretiens, les conseillers se sont exprimés sur leur représentation du travail des éleveurs, la place de la thématique travail dans leur activité de conseil, leurs pratiques de conseil, leurs réussites et difficultés, leur connaissance des actions menées localement sur le travail, et leurs propositions quant aux améliorations à apporter dans l'accompagnement des éleveurs. Les éleveurs ont été questionnés sur leurs propres représentations du travail, leurs aspirations en termes d'évolution de leur situation, leur perception de l'action qui les a concernés, les changements qui en ont résulté dans leur métier ou leur vie personnelle, et leurs attentes pour la suite.

De façon moins approfondie, six expériences de terrain (Kling-Eveillard et al 2008) ont fait l'objet d'une analyse à partir de documents écrits (recueil de données factuelles) et de quelques entretiens en face-à-face ou par téléphone avec les animateurs d'organismes de développement et des éleveurs (recueil de leur expérience et de leur ressenti). Enfin, une soixantaine d'expériences de terrain (Kling-Eveillard et al 2007) ont été recensées et décrites par écrit (type d'action, objectifs opérationnels, public, partenaires, durée prévue, état d'avancement) par les personnes chargées de les mettre en œuvre (le plus souvent des conseillers de terrain) qui ont formulé les points forts et les difficultés rencontrées et parfois leurs propositions pour améliorer le conseil sur le travail.

En complément, d'autres sources ont été mobilisées afin de valider les hypothèses sur les difficultés rencontrées par les conseillers et d'enrichir les recommandations. Il s'agit d'avis et de témoignages recueillis à l'occasion de trois ateliers (de vingt participants environ chacun) organisés pendant les rencontres nationales de Rennes du RMT Travail en 2009. Ils réunissaient des conseillers confrontés dans leur activité à l'accompagnement des éleveurs sur le travail. Chaque atelier portait sur un thème en lien avec le conseil et, après deux ou trois interventions introductives, proposait de débattre pour dégager les questions clés et des recommandations pour le conseil (Fiorelli et Couzy 2009, Gédouin et Guillaumin 2009, Tellier et Chauvat 2009).

L'ensemble de ces données, études et avis de conseillers, permet d'identifier plusieurs contextes différents (voir en particulier Rubin et Sabatté 2008, Sabatté et Huchon 2009, Renard 2009) dans lesquels ils interviennent en élevage sur la question du travail. Ces contextes, que nous décrivons dans cet article sans prétendre à l'exhaustivité, permettent de structurer la diversité des façons dont les conseillers s'engagent dans le traitement de la question du travail. Ainsi, certains disent aborder le travail dans leur activité habituelle, dans le cadre d'un conseil technique. Ils l'intègrent dans leur approche de la conduite d'élevage. Ils peuvent considérer comme important que les conditions de travail ne se trouvent pas dégradées par des changements techniques envisagés dans l'exploitation. Ils proposent ainsi d'aborder, par exemple dans un conseil sur les mammites, la répartition des responsabilités et des tâches pour leur détection au moment de la traite. Dans d'autres cas, les aspects travail peuvent être abordés à l'occasion de la réflexion autour d'un projet, et le travail peut constituer une des dimensions étudiées sans pour autant en être l'objet central. Il peut s'agir par exemple d'un projet de bâtiment, d'une évolution du système de production, ou encore d'une évolution des activités. L'éleveur est accompagné sur son projet par un conseiller d'entreprise ou par un conseiller spécialisé comme un conseiller bâtiment par exemple. Le travail est alors traité comme l'un des éléments d'une exploration plus large portant sur les finalités poursuivies par l'éleveur et chacun des membres du collectif, sur leurs satisfactions et insatisfactions, sur ce qu'ils souhaitent voir évoluer. Enfin, l'activité de conseil peut être centrée sur le travail, à l'occasion, par exemple, de la recherche par l'éleveur d'une amélioration des conditions de travail ou de la qualité de vie, à partir d'un constat que «ça ne peut pas continuer comme ça» et qui constitue le point de départ d'une réflexion.

Dans ces différents contextes, les conseillers ne développent pas la même approche du travail et la mise en œuvre concrète du conseil dépend également des missions données aux conseillers et de la manière dont les organismes de conseil sur un territoire s'organisent pour construire une offre de conseil sur le travail en élevage. Notre étude vise à comprendre comment, de façon transversale à ces contextes, émergent des difficultés dans la mise en œuvre d'un conseil sur le travail.

Nos résultats portent tout autant sur les difficultés rencontrées par les conseillers que sur ce qu'ils ont tenté ou sur ce qu'ils suggèrent à partir de leur expérience pour les lever ou les contourner, afin d'identifier des pistes de recommandations pour la mise en œuvre d'un conseil sur le travail en élevage.

\section{3 / Le travail : source de dif- ficultés spécifiques dans le conseil}

Le cadre d'analyse que nous avons choisi fait ressortir qu'une partie des difficultés associées à la mise en œuvre d'un conseil sur le travail sont largement liées à la spécificité de ce thème. D'autres présentent un caractère plus général et rejoignent celles rencontrées dans tout accompagnement d'agriculteurs dans le changement.

Les difficultés qui renvoient au niveau technique sont en particulier en relation avec les représentations des acteurs sur un sujet à la fois intime et tabou. Celles en lien avec le niveau interactionnel ou contractuel sont plutôt dues au caractère multifacette du sujet du travail. Ainsi, ce caractère renvoie, au niveau interactionnel, à la question de la posture du conseiller et à son influence sur ce qui se construit et se joue entre le conseiller et l'éleveur dans la situation de conseil. Au niveau contractuel, les questions portent sur le repérage et l'organisation des compétences pour permettre, quand cela apparaît nécessaire, un passage de relais d'un conseiller vers un autre mieux placé pour accompagner l'éleveur.

\section{1 / Le travail, un sujet intime et tabou}

$\mathrm{Au}$ niveau technique, les conseillers mettent en avant le caractère intime 
mais aussi tabou du travail dans les études de Kling-Eveillard et al (2007) et de Renard (2009).

Un sujet intime tout d'abord, parce que, selon les conseillers, il ne concerne pas que l'exploitation ou qu'un aspect de la conduite ou du système, mais qu'il renvoie à la personne, son identité, ses conditions de vie, ses aspirations, son projet de vie personnel et familial et, qu'en cela, il met au centre des préoccupations les aspects humains. L'intimité en jeu est également celle du collectif de travail, familial ou non, avec ses relations propres, ses conflits éventuels.

Les conseillers eux-mêmes sont porteurs de jugements de valeur sur les éleveurs, dépréciant parfois ceux qui, de leur point de vue, «ne se fatiguent pas». Le travail reste en effet une valeur forte dans le monde agricole. Ce caractère intime et tabou du travail crée ainsi une difficulté pour les éleveurs à en parler et pour les conseillers à l'aborder avec eux, à entendre, accueillir ou susciter une demande éventuelle d'appui sur ce sujet.

Face à ce sujet qu'ils qualifient de «délicat», de nombreux conseillers interrogés font part tout à la fois de leur appréhension à soulever ces questions, de leur méconnaissance du sujet du travail et de ses contours, et de leur manque de compétences pour l'aborder.

Des pistes émergent des retours de terrain pour dépasser ces difficultés. Ainsi, dans les travaux de KlingEveillard et al (2008) et de Warcoin (2010) la mise en main de questionnaires de type autodiagnostic, le plus souvent courts, appelés «brise-glaces», qui favorisent l'expression des éleveurs, est appréciée des conseillers peu expérimentés sur le sujet. Quel que soit l'outil, serait-on tenté de dire, du moment qu'il existe, l'essentiel est de disposer d'un support pour la discussion, offrant la possibilité de faire le tour de plusieurs facettes du sujet, et permettant aussi de rassurer le conseiller. Il instrumentalise le questionnement et le rend légitime aux yeux des éleveurs comme des conseillers. Ceux qui utilisent un tel outil reconnaissent fréquemment l'adapter à leurs besoins (Renard 2009).

De même, la formation proposée aux conseillers dans certaines actions est reconnue comme utile voire indispensable. Ainsi, ceux interrogés dans l'évaluation de l'action «Paqvel» (Roussez 2008) qui ont eux-mêmes été formés avant d'intervenir auprès des éleveurs soulignent l'importance d'être sensibilisés aux différentes dimensions du travail, et pas seulement formés à la prise en main d'un outil pour le diagnostic ou le conseil.
Dans l'atelier animé par Fiorelli et Couzy (2009), certains conseillers reconnaissent être à l'aise pour aborder le sujet avec un éleveur tout en le qualifiant également d'intime et tabou. Ils évoquent souvent une sensibilité personnelle qui les a conduits à s'intéresser et à mettre en œuvre ce type d'approche. Ils insistent sur l'utilité d'un outil et de la formation. D'autres évoquent un nécessaire travail personnel sur soimême, sur ses propres représentations et son vécu du travail avant d'aborder le sujet avec les éleveurs dans le conseil.

A partir des travaux de Marty (2004), Roussez (2008), Kling-Eveillard et al (2007, 2008, 2010), nous pouvons faire le constat que de nombreux conseillers mettent en avant l'intérêt de l'animation de groupes d'éleveurs pour accompagner une réflexion sur le travail. Le groupe facilite, selon eux, la prise de parole sur le travail, suscite chez les participants un recul par rapport à ce qu'ils vivent et l'expression de leurs souhaits ou de leurs difficultés, tout en les rassurant puisqu'ils les partagent avec d'autres éleveurs dans des situations comparables. La confrontation des expériences et des idées favoriserait le changement d'attitudes et les témoignages de pairs sont toujours plus convaincants et parlants qu'un apport théorique fait par un conseiller. Le groupe débouche même parfois sur des solutions collectives (par exemple par la mise en commun de matériel ou de main-d'œuvre) quand il est constitué à une échelle locale. Si les animations de groupes d'éleveurs facilitent indéniablement l'expression de leurs problèmes d'organisation du travail, il demeure cependant difficile de les convaincre de participer à ces sessions collectives.

\section{2 / Le travail, un sujet multifa- cette}

Les conseillers disent fréquemment dans les enquêtes (Marty 2004, Roussez 2008, Renard 2009, Warcoin 2010) que le sujet du travail en élevage est complexe. Ils le décrivent soit en renvoyant à la façon dont le travail est qualifié (ressource, combinaison d'activités ou expression de rationalités subjectives), soit aux différentes dimensions pour l'appréhender (volume de travail, répartition dans l'année, relations entre les membres du collectif...) et parfois aux différentes disciplines qui peuvent apporter un éclairage (zootechnie, sociologie, ergonomie...). La complexité des questions sur le travail requiert bien souvent une approche globale de l'exploitation et pas seulement d'un atelier ou d'une thématique, ainsi que la prise en compte de l'ensemble des personnes qui y travaillent quels que soient leurs statuts.
Ce caractère multifacette a, selon les conseillers interrogés dans nos études, deux implications importantes dans le conseil. D'une part, il intervient dans le choix d'une posture pour accompagner l'éleveur en fonction de la facette abordée et d'autre part, il mobilise une palette de compétences nécessitant souvent le recours à plusieurs intervenants.

a) Savoir choisir une posture selon la dimension du travail abordée et selon l'avancement du conseil

La demande sur le travail peut être directement formulée par l'éleveur en termes d'attente d'une solution sur un aspect précis de la conduite ou de l'organisation. Dans d'autres cas, les éleveurs évoquent plutôt des difficultés et des insatisfactions vis-à-vis de la situation actuelle sans pour autant qu'elles soient explicitement formulées en terme de travail. Enfin, ce sont quelquefois les conseillers eux-mêmes qui détectent un problème chez un éleveur et qui s'emploient alors à leur proposer un conseil sur la thématique du travail.

Face à ces situations très différentes, les conseillers interrogés dans nos études disent avoir à choisir une posture, entre une réponse ponctuelle ou globale, entre l'approche par la technique ou la prise en compte des personnes, entre l'entrée par une dimension du travail ou par plusieurs. Dans certains cas, le conseiller se positionne comme un prescripteur ou un expert, qui apporte des références et des connaissances en réponse à une question explicite ou implicite. Dans d'autres, il joue un rôle d'accompagnateur, capable de favoriser l'expression par l'éleveur de ses objectifs et de ses aspirations. Il est alors présent tout au long du processus, depuis la formulation du problème jusqu'à la réflexion sur les solutions, voire à leur mise en œuvre effective (Rubin et Sabatté 2008, Renard 2009, Tellier et Chauvat 2009).

Là encore, l'expérience de certains conseillers fournit des pistes de recommandations sur cette question de la posture. Pour eux, la posture est à réfléchir et à choisir effectivement en fonction du contexte dans lequel le travail est abordé (par exemple un conseil technique ou un conseil centré sur le travail), de la demande telle qu'elle est exprimée ou non, mais également de leurs propres compétences. La posture pertinente peut, de plus, être différente selon l'avancement de l'accompagnement. Le recours à l'expertise technique du conseiller s'avère par exemple nécessaire dans certaines situations au moment d'envisager des modifications de la conduite ou de l'organisation en vue de l'amélioration. Un même conseiller 
peut ainsi être amené à adopter alternativement une posture d'accompagnateur et une posture d'expert technique.

Les conseillers mettent l'accent sur trois éléments utiles à mobiliser dans l'une ou l'autre des postures de conseil (Kling-Eveillard et al 2008, Fiorelli et Couzy 2009, Renard 2009, Sabatté et Huchon 2009) :

- L'écoute active. Il s'agit d'une écoute de ce qui n'est pas toujours spontanément exprimé mais est latent derrière par exemple des difficultés ponctuelles. Un questionnement de type ouvert permet de recueillir les représentations sociales et les points de vue de la personne concernée et d'éviter de l'influencer par sa propre vision ;

- Une prise en compte des personnes, de l'humain, dans leur vécu, leurs aspirations, leurs plaisirs et déplaisirs au travail, leurs rationalités subjectives ;

- La co-construction par l'éleveur et le conseiller de la problématique à traiter et du plan d'amélioration, qui constitue tout à la fois un processus nécessitant du temps, des compétences et un cadre nouveau pour le conseil, adapté pour ce sujet complexe et global.

Ces trois éléments ne sont pas spécifiques du conseil sur le travail, ils peuvent également être mobilisés dans le conseil sur d'autres thématiques, en particulier dans un accompagnement stratégique. Les conseillers qui n'abordent que depuis peu le sujet du travail les évoquent cependant spécifiquement à propos du conseil sur le travail car il les amène à se questionner sur leur mode $\mathrm{d}$ 'intervention et à mobiliser certains de ces éléments, nouveaux pour eux, voire à se former sur certains si besoin (Tellier et Chauvat 2009).

b) Repérer et organiser des compétences et des intervenants

Face à la diversité des manières dont les éleveurs expriment leur demande et à la diversité des facettes du travail qui en font l'objet, les conseillers confrontés au conseil sur le travail constatent l'impossibilité pour une seule personne, voire même pour un seul organisme, de répondre à toutes les interrogations des éleveurs, et insistent sur la nécessité de structurer une offre de conseil qui articule des compétences diverses et permette le passage de relais si besoin en fonction des compétences de chacun. Ce constat ressort nettement des avis exprimés à l'occasion du recensement de soixante actions d'accompagnement sur le travail mis en forme par KlingEveillard (2007).

Cette difficulté touche le niveau contractuel: les conseillers question- nent le dispositif (pris au sens large) qui lie l'éleveur à l'offre de service dans sa capacité à traiter le caractère multifacette du travail.

Des freins à la mise en œuvre d'un partage de compétences à l'échelle d'un territoire sont évoqués par les conseillers enquêtés: tout d'abord la méconnaissance des autres structures, des compétences et des intervenants potentiels sur le travail en élevage, ensuite l'absence de coordination et d'organisation entre les structures et enfin l'existence de situations de concurrence entre certains organismes.

Dans les actions de terrain analysées dans nos études, les solutions organisationnelles pour cette articulation des compétences prennent différentes formes. En Pays de la Loire (Rubin et Sabatté 2008, Sabatté et Huchon 2009), des passages de relais se produisent dans un cadre interne à une structure, entre des collègues de profils, activités et compétences différents et identifiés. Ainsi, un conseiller de Chambre d'Agriculture interlocuteur habituel de l'éleveur, endosse le rôle de référent ou d'accompagnateur de l'éleveur et sollicite en interne un conseiller bâtiment, un conseiller d'entreprise, ou encore une personne chargée des questions d'emploi en agriculture. Dans d'autres situations il s'agit de relais avec des partenaires extérieurs, par exemple la Mutualité Sociale Agricole (MSA) pour traiter des questions de santé ou sécurité au travail. Les conseillers précisent souvent que les conditions économiques des organismes de développement ne s'y prêtent pas toujours facilement ni spontanément surtout si ces services se monnaient auprès des éleveurs (Sabatté et Huchon 2009).

Comme évoqué plus haut, les conseillers sont souvent demandeurs d'une coordination formalisée des intervenants avec un schéma d'articulation explicite. Ils sont aussi intéressés par l'animation du réseau des intervenants qui se rencontrent pour mieux se connaître, se forment, échangent sur leurs pratiques, co-construisent des méthodes et des outils d'intervention (KlingEveillard et al 2008, Gédouin et Guillaumin 2009, Sabatté et Huchon 2009, Renard 2009, Kling-Eveillard et al 2010). Enfin, ils font état d'une nécessaire implication des responsables professionnels garante de la reconnaissance de leur légitimité dans l'exercice d'un conseil sur le travail (Renard 2009).

\section{3 / Le travail, une question à traiter dans la durée}

Du fait du caractère à la fois intime et multifacette du travail, le conseil sur ce thème nécessite du temps (Renard 2009). Il apparaît difficile aux conseillers d'amorcer une réflexion sur le thème du travail et d'accompagner l'éleveur en un seul passage sur l'exploitation vers des solutions qui pourront impliquer de fortes remises en cause de son organisation et de ses façons de faire. Tant que les modifications envisagées ne concernent que la technique de production ou une pratique d'élevage, la décision peut être prise rapidement suite au passage d'un conseiller. Sa mise en pratique dépend alors uniquement du processus de production et du temps nécessaire à sa mise en œuvre. Conseiller le travail d'un éleveur peut aussi amener à des remises en cause des objectifs, des pratiques, de l'organisation, et demander du temps pour la réflexion et la prise de conscience sur ses difficultés et ses priorités, et également pour la maturation des bonnes décisions à prendre pour améliorer la situation.

A titre d'exemple, en Pays de la Loire, l'accompagnement d'éleveurs laitiers par un binôme pluridisciplinaire s'est étalé sur près d'un an (340 jours) en moyenne depuis l'identification de la problématique jusqu'à la mise en œuvre de solutions, avec entre temps trois rencontres en moyenne entre les intervenants et l'éleveur (Rubin et Sabatté 2008).

Le cadre de la prestation et son coût doivent pouvoir s'adapter à la prise en compte de la dynamique de changement si plusieurs passages du conseiller dans l'exploitation espacés dans le temps s'avèrent nécessaires. C'est parfois difficile de le prévoir et donc de calibrer la prestation à l'avance.

\section{4 / A chaque étape d'un conseil sur le travail, ses difficultés}

Les conseillers interrogés sont nombreux à dire que la première étape d'émergence de la demande est délicate (Kling-Eveillard et al 2007, KlingEveillard et al 2008, Gédouin et Guillaumin 2009). Entendre une demande d'accompagnement ou faire émerger des difficultés en termes de travail est possible quand le conseiller maîtrise suffisamment le sujet du travail et se sent légitime et compétent pour le faire. C'est plus difficile pour des conseillers à profil technique qui appréhendent le travail comme une ressource ou un facteur de production mais qui n'en connaissent pas ou n'en maîtrisent pas les autres facettes.

Dans cette étape, les difficultés portent sur deux des trois niveaux du cadre 
d'analyse de la relation de conseil. Le caractère intime et tabou du sujet du travail (niveau technique) rend plus difficile que pour d'autres sujets en élevage l'expression d'une demande par les éleveurs et sa prise en compte par le conseiller. Le niveau contractuel intervient en fournissant ou non au conseiller la marge de manœuvre, et en particulier le temps et la légitimité pour accueillir et relayer si nécessaire auprès d'autres intervenants une demande d'éleveur sur le travail.

Une fois la demande accueillie et identifiée comme étant en lien avec le travail, les conseillers évoquent souvent la difficulté à passer des insatisfactions exprimées par l'éleveur à une problématique qui pourra être traitée dans le conseil, ce que certains appellent «dépasser la plainte». Nous retrouvons, dans cette étape de diagnostic, le niveau interactionnel de la relation, puisqu'il s'agit de posture. En particulier, quand un accompagnement approfondi apparaît nécessaire, cela peut amener l'éleveur et le conseiller à s'engager dans une co-construction de la problématique à traiter dans le conseil.

Dans l'étape de développement des solutions, le niveau contractuel de la relation est en jeu puisque les difficultés évoquées par les conseillers interrogés portent essentiellement sur la mobilisation de compétences complémentaires qui apparaissent nécessaires.

Les compétences des conseillers constituent un élément décisif pour identifier l'existence d'un problème de travail, comprendre la demande d'un éleveur, aider à la formulation du problème et orienter, soit vers une solution ponctuelle, soit vers un accompagnement approfondi. Cela passe en particulier par l'acquisition de connaissances sur les différentes facettes du sujet, par la maîtrise de méthodes et d'outils (d'expression des aspirations, de diagnostic...) et le recours à des références.

La conception de ces méthodes et outils peut être un moyen de sensibiliser et former les intervenants à la question du travail. Lorsque l'élaboration est collective et implique plusieurs conseillers, cela favorise la pertinence par rapport au terrain et l'appropriation. La phase de prise en main de l'outil par les intervenants en élevage apparaît comme une étape déterminante pour son utilisation effective dans le conseil (Renard 2009, Warcoin 2010). On constate qu'elle passe par des formations qui abordent la problématique du travail dans son ensemble et pas seulement l'outil, et qui ont un caractère pratique pour prendre en compte le contexte concret des futurs utilisateurs comme dans l'action «Vip» (Kling-Eveillard et al 2010).

\section{5 / Discussion - Conclusion}

L'analyse des difficultés rencontrées par les conseillers met en évidence que l'émergence d'un besoin ou d'une demande d'accompagnement sur le sujet du travail est délicate à la fois du côté des éleveurs et du côté des conseillers qui ont souvent du mal à l'accueillir ou à la susciter. Pour les éleveurs, ceci trouve sans doute sa source dans le fait que le travail est un sujet très personnel qui renvoie à l'identité, à l'image de soi et à ses valeurs. Pour les conseillers, nous suggérons qu'ils sont peu préparés à prendre en compte plus particulièrement certaines des rationalités subjectives décrites par Fiorelli et al (2010), en particulier la rationalité identitaire (son identité en tant qu'individu et éleveur), la rationalité relationnelle (avec les personnes et les animaux) et la rationalité de l'engagement du corps au travail, et cela d'autant plus pour les conseillers qui ont un profil ou une approche plutôt technique. Il est également possible qu'ils éprouvent une difficulté à inscrire leur intervention dans l'ensemble des facteurs socioculturels qui, d'après Merin (2003), sont en jeu dans un conseil sur le travail et l'emploi en milieu rural, comme ils ont également une difficulté à s'ajuster, ainsi que le souligne Compagnone (2006), à l'univers cognitif des agriculteurs dans l'interaction.

Au-delà de la compétence, Lémery (2006) fournit une piste d'explication en décrivant le conseiller agricole soumis à deux injonctions contradictoires, d'un côté une fonction d'aide à la résolution de problèmes dans un contexte d'incertitude et de complexité croissante, et de l'autre une fonction de contrôle, d'appui réglementaire et administratif. Les conseillers se trouveraient ainsi en recherche de repères, de légitimité et de rôle, et cela peut s'avérer présent dès lors qu'il s'agit à la fois de conseiller sur le travail et de faire valoir les règles du droit du travail par exemple.

En raison de ses multiples dimensions, le travail peut être abordé dans le conseil de façon très diverse selon le type de demande et son degré de formulation. Ceci implique, pour le conseiller, de saisir s'il peut répondre seul à la demande, ou s'il doit rechercher d'autres compétences, d'autres profils voire d'autres approches ou disciplines. Cette difficulté, mais aussi cette nécessité de concevoir des interventions «multiacteurs» ont également été relevées par Merin (2003) concernant le cadre de l'intervention ergonomique pour amé- liorer une situation vis-à-vis du travail. Nous avons suggéré que le cadre organisationnel dans lequel les conseillers exercent leur activité peut permettre de faciliter le repérage et l'organisation des compétences.

A ces difficultés, qui nous semblent liées à la fois au caractère intime, tabou mais aussi multifacette du travail, s'ajoute celle qui renvoie à la nécessité de choisir entre différentes postures, avec ce que chacune implique. Plusieurs auteurs constatent effectivement que les compétences des conseillers évoluent vers, ou intègrent de plus en plus des compétences d'animation plus larges (Compagnone et Lémery 2009), voire la co-construction (Cerf et Maxime 2006), tout en soulignant les difficultés éventuelles que les conseillers éprouvent à se glisser dans ces nouvelles postures. Ainsi, certains aspects évoqués par les conseillers sur le travail en élevage se retrouvent dans tout accompagnement stratégique qui mobilise une approche globale de l'exploitation ainsi que les techniques d'écoute active (voir par exemple Cerf et Maxime 2006). Le conseil stratégique comme le conseil sur le travail peuvent aboutir à des remises en cause des choix d'orientation, de conduite et d'organisation, mais aussi viennent questionner directement en tant que personne celui ou celle qui est au cœur de la démarche d'accompagnement.

Ainsi, la prise en compte du travail dans le conseil apparaît alors comme une occasion de questionnement des conseillers sur leur rôle, leurs pratiques et leurs compétences, et s'inscrit bien dans les tendances en cours de l'évolution du conseil en agriculture.

Face à ces difficultés, en nous appuyant aussi sur les pistes évoquées par les conseillers eux-mêmes, quelques recommandations peuvent être formulées autour de deux pôles complémentaires: le conseiller lui-même, et le dispositif qui instaure le cadre opérationnel pour l'accompagnement.

D'un côté, il apparaît nécessaire de permettre aux conseillers de faire évoluer leurs connaissances et leurs compétences et de leur fournir des méthodes et des outils qui leur permettent en quelque sorte de «dédramatiser» le sujet pour le rendre traitable et discutable.

De l'autre, il est nécessaire que le dispositif, tel que cette notion est définie par Renard (2009), mette en avant un schéma de fonctionnement définissant les formes d'interaction et de coordination (Compagnone et Lémery 2009), et plus particulièrement, précise les modes d'articulation entre des per- 
sonnes et des organismes différents à l'échelle d'un territoire, ce qu'évoquent également Auricoste et al (2011). Un tel cadre est de plus garant du respect de la durée d'un accompagnement sur le thème du travail, paramètre important pour conduire la réflexion sur la situation et le vécu, le diagnostic et la maturation d'un plan d'amélioration.

Une autre évolution de l'activité agricole pointée par Auricoste et al (2011), appelée désectorisation partielle, et qui correspond à l'implication d'acteurs ne se définissant pas principalement par rapport à l'agriculture, se trouve également à l'œuvre sur le sujet du travail, quand par exemple un ergonome habitué aux entreprises industrielles est associé à un projet centré sur l'élevage et développe une méthode d'observation de l'éleveur dans son travail.

Néanmoins, la mise en œuvre concrète de ces recommandations se heurte à des limites de modalités d'intervention, de coût, de coordination entre des structures parfois concurrentes et dont les agents ne se connaissent pas. Le thème du travail est, de plus, peu mis en avant dans les politiques institutionnelles et de fait recueille peu de moyens techniques et financiers.

Nos premières analyses et recommandations sont à affiner, en développant d'autres travaux pour en valider les conclusions. Ceci passe certainement par un nouveau travail de capitalisation d'expériences de terrain, d'évaluation des effets, tant en termes de satisfaction des acteurs que d'impact du conseil au niveau des éleveurs, puisqu'au bout du compte c'est bien l'amélioration de la situation des éleveurs qui est visée à travers ces accompagnements et qui doit être appréciée. Il pourrait s'agir en particulier de s'intéresser à la dynamique de la prise en compte du travail dans le conseil, depuis des accompagnements centrés sur le travail (par exemple autour des collectifs de travail, du salariat, de la transmission, de la réponse aux aspirations en termes de temps libéré) jusqu'à une intégration du sujet travail dans tout conseil comme un des angles de l'analyse.

\section{Remerciements}

Nous remercions pour leur contribution à nos réflexions : Pascal Bouchart (ELC3 Picardie), Caroline Depoudent (Chambre d'Agriculture de Bretagne), Anne-Charlotte Dockès et Brigitte Frappat (Institut de l'Elevage), Guy Jauneau (Chambre d'Agriculture de l'Isère), Madeleine Jélu (Chambre d'Agriculture de la Mayenne), Nicolas Lion (Chambre d'Agriculture de l'Aisne), Christophe Vannier (Chambre d'Agriculture du Maine-et-Loire), Claudie Visière (Fncuma).

\section{Références}

Auricoste C., Albaladejo C., Barthe L., Couix N., Duvernoy I., Girard N., Gross H., Labatut J. Lenormand P., 2011. Accompagner l'activité agricole dans les territoires : au carrefour entre le développement sectoriel et le développement territorial. Cah. Agric., 20, 395-412.

Béguin P., Dedieu B., Sabourin E., 2011 Introduction. In : Le travail en agriculture : son organisation et ses valeurs face à l'innovation. Béguin P., Dedieu B., Sabourin E. (Ed) Editions L'harmattan, Paris, France, 11-16.

Blanchet A., Gotman A., 1992. L'enquête et ses méthodes : l'entretien. Nathan Université, Paris, France, 128p.

Cerf M., Falzon P., 2005. Le client dans la relation. In : Situations de services : travailler en interaction. Cerf M., Falzon P. (Eds). Editiosn PUF, série Travail Humain, 41-60.

Cerf M., Maxime F., 2006. La coproduction $\mathrm{du}$ conseil: un apprentissage difficile. In : Conseiller en agriculture. Rémy J., Brives H., Lémery B. (coord). INRA Educagri Editions, Dijon, France, 137-152.

Compagnone C., 2006. Le juste dans la relation de conseil en agriculture. In : Conseiller en agriculture. Rémy J., Brives H., Lémery B. (coord). INRA Educagri Editions, Dijon, France, 221-234.

Compagnone C., Lémery B., 2009. Conclusion. In : Conseil et développement en agriculture. Compagnone C., Auricoste C., Lémery B. (coord). INRA Educagri Editions, Dijon, France, 235-242.

Couzy C., Dockès A.C., 2006. Multiplicité des métiers, diversité des modèles de référence: un éclairage sur les transformations des métiers des agriculteurs. Renc. Rech. Rum., $13,51-54$.

Dedieu B., Servière G., 2011. Les modèles du travail en élevage: points de vue de zootechniciens des systèmes d'élevage. In : Le travail en agriculture: son organisation et ses valeurs face à l'innovation. Béguin P., Dedieu
B., Sabourin E. (Ed). Editions L'Harmattan, Paris, France, 155-169.

Fiorelli C., Couzy C., 2009. Atelier $n^{\circ} 7$ Aborder le travail : partage d'expériences sur les façons d'être. In : 3èmes Rencontres nationales travail en élevage - Recueil des contributions. Institut de l'Elevage. 137-144. http://idele.fr/domaines-techniques/sequiperet-sorganiser/travail-en-elevage/publication/ idelesolr/recommends/atelier-7-des-3e-rencontres-travail-en-elevage-aborder-le-travail-partage-dexperiences-sur.htm

Fiorelli C., Dedieu B., Porcher J., 2010. Un cadre d'analyse des compromis adoptés par les éleveurs pour organiser leur travail. Cah. Agric. $19,5,383-390$

Gédouin M, Guillaumin A., 2009. Atelier $\mathrm{n}^{\circ} 3$ : Actions partenariales d'accompagnement des éleveurs: quelle organisation pour être efficace ? In : $3^{\text {emes }}$ Rencontres nationales travail en élevage - Recueil des contributions. Institut de l'Elevage. 101-106. http://idele.fr/domaines-techniques/sequiperet-sorganiser/travail-en-elevage/publication/ idelesolr/recommends/atelier-3-des-3e-rencontres-travail-en-elevage-actions-partenarialesdaccompagnement-des-ele.html

Ghiglione R., Matalon B., 2008. Les enquêtes sociologiques, théories et pratique. 6ème édition. Collection U. Editions Armand Colin, Paris, France, 301p.

Goffman E., 1968. Asiles : études sur la condition sociale des malades mentaux et autres reclus. Collection «Le sens commun». Editions de Minuit, Paris, France, 452p.

Kling-Eveillard F., Dedieu B., Chauvat S., Servière G., 2007. Recensement d'expériences autour du travail en élevage conduites par le développement, la recherche et la formation. Collection Résultats. Institut de l'Elevage, Paris, France, 42p.

Kling-Eveillard F., Dedieu B., Frappat B. Chauvat S., Servière G., 2008. Compte rendu de six expériences locales d'accompagnement des éleveurs sur le travail. Collection Résultats. Institut de l'Elevage, Paris, France, 54p.

Kling-Eveillard F., Gédouin M., Béguin E., Machefer J., 2010. Une action régionale pour accompagner les éleveurs sur le travail: l'exemple de «vivre l'élevage en Picardie». In : Actes Colloque SFER «Conseil en agriculture acteurs, marchés, mutations», Dijon, France. http://www.sfer.asso.fr

Lémery B., 2006. Nouvelle agriculture, nouvelles formes d'exercice et nouveaux enjeux du conseil aux agriculteurs. In: Conseiller en agriculture. Rémy J., Brives H., Lémery B. (coord). INRA Educagri Editions, Dijon, France, 235-252

Marty M., 2004. Evaluation d'une opération régionale d'accompagnement des producteurs de lait sur thème du travail en élevage bovin lait. Mémoire de fin d'études ESA Purpan. $233 p$.

Méda D., 2007. Le travail. 3 ème édition mise à jour. Collection Que sais-je ? PUF Editions, Paris, France, $127 \mathrm{p}$

Merin S., 2003. L'analyse ergonomique du travail interpelée par la prise en compte de facteurs socioculturels. In: Actes du 38 eme Congrès de la SELF : Modèles et pratiques de l'analyse du travail, 15 ans d'évolution. Paris, France, 487-492.

Renard F., 2009. L'analyse de la mise en œuvre d'outils de conseil sur le travail en élevage. Mémoire de fin d'études Agrocampus Rennes, France, 97p.

Roussez P., 2008. Evaluation de l'action régionale PAQVEL. Mémoire de fin d'études, Esitpa, 92p.

Rubin B., Sabatté N., 2008. Une approche pluridisciplinaire de conseil «travail» en élevage laitier, synthèse de quinze expériences en Pays de la Loire. Collection Résultats. Institut de l'Elevage, Paris, France, 70p. 
Sabatté N., Huchon J.C., 2009. Accompagner les éleveurs sur le travail: Réflexions sur le métier de conseiller. In : 3 emes Rencontres nationales travail en élevage - Recueil des contributions. Institut de l'Elevage, Paris, France, 31-36.

Tellier P., Chauvat S., 2009. Atelier $n^{\circ} 4$ : Evolution du métier de conseiller : de l'identi- fication des besoins à l'accompagnement vers un changement des conditions de travail. In 3 èmes Rencontres nationales travail en élevage - Recueil des contributions. Institut de l'Elevage. 107-116. http://idele.fr/domainestechniques/sequiper-et-sorganiser/travail-enelevage/publication/idelesolr/recommends/atelier-4-des-3e-rencontres-travail-en-elevage-evo $\underline{\text { lution-du-metier-de-conseiller-de-la-pres.html }}$

Warcoin C., 2010. Evaluation à mi-parcours du programme Vivre l'Elevage en Picardie. Mémoire de fin d'études Institut Lasalle Beauvais, France, 111p.

\title{
Résumé
}

De façon paradoxale, alors que le travail constitue un enjeu important pour la pérennité des exploitations d'élevage, les éleveurs en parlent relativement peu et sollicitent rarement de façon spontanée un accompagnement sur ce thème. Parallèlement, les conseillers sont nombreux à faire part de leurs difficultés à s'approprier le sujet et à se positionner comme des personnes ressources sur ce thème auprès des éleveurs. A partir d'expériences de terrain, nous avons analysé les difficultés que les conseillers ressentent et expriment, en vue de formuler quelques recommandations pour les lever. Parmi les difficultés énoncées, certaines sont liées au fait que le travail est à la fois un sujet intime et tabou, et un sujet multifacette. Ces caractéristiques questionnent d'une part le choix d'une posture adéquate (prescription, expertise, accompagnement...) selon la situation de conseil dans laquelle ce sujet est abordé, et d'autre part les relations avec les collègues, car il est souvent nécessaire de s'appuyer sur des compétences et des intervenants complémentaires. Les recommandations pour la mise en œuvre d'un conseil sur le travail portent sur les évolutions des pratiques des conseillers eux-mêmes mais aussi sur le cadre organisationnel. Les conseillers ont tout d'abord besoin de connaissances sur le champ du travail, de compétences pour faciliter l'émergence de besoins, accompagner dans la durée les agriculteurs, et ont besoin d'outils et de méthodes pour étayer leur conseil. Le cadre organisationnel doit aider à articuler des compétences et des organismes complémentaires afin de constituer une offre de conseil centrée sur le travail.

\begin{abstract}
Working conditions on livestock farms, a personal and multifaceted topic: some recommendations to help farmers' advisers

On the one hand, working conditions constitute a major concern for livestock farmers. On the other hand, farmers rarely raise this issue nor ask for advice about it. In parallel, many advisers find it difficult to provide services to farmers on this topic. We analyzed the difficulties they meet through several field experiences, in order to formulate recommendations that could help to better support farmers. Some difficulties are linked to the personal and multifaceted characteristics of the topic. This aspect has to be taken into account by advisers when choosing their position towards farmers (technical expert, facilitator...), depending on the moment and the situation. Other troubles refer to the collaboration with colleagues, since additional skills or knowledge must be solicited to treat working issues . To improve the situation, we point out that it is essential to help advisers to adapt their practices and positioning, to increase their knowledge of the work on livestock farms, to gain new skills, approaches and tools to support farmers. The organizational framework also has to be worked so that complementary work involving several organizations and players is possible.
\end{abstract}

KLING-EVEILLARD F., CERF M., CHAUVAT S., SABATTE N., 2012. Le travail, sujet intime et multifacette : premières recommandations pour l'aborder dans le conseil en élevage. In : Numéro spécial, Travail en élevage. Hostiou N., Dedieu B., Baumont R. (Eds). INRA Prod. Anim., 25, 211-220. 